\title{
Galaxy Evolution in Three dimensions : Time, Space and Mass
}

\author{
Tadayuki Kodama ${ }^{1}$, Richard Bower ${ }^{2}$, Philip Best ${ }^{3}$, Patrick Hall ${ }^{4}$, Toru \\ Yamada $^{1}$, and Masayuki Tanaka ${ }^{5}$ \\ 1 National Astronomical Observatory of Japan, 2-21-1 Osawa, Mitaka, Tokyo, \\ 181-8588, Japan \\ 2 Department of Physics, University of Durham, South Road, Durham DH1 3LE, UK \\ 3 Institute for Astronomy, Royal Observatory Edinburgh, Blackford Hill, Edinburgh \\ EH9 3HJ \\ 4 Department of Astrophysical Sciences, Princeton University, Peyton Hall, Ivy Lane, \\ Princeton, NJ 08544-1001, USA \\ 5 Department of Astronomy, School of Science, University of Tokyo, Bunkyo-ku, \\ Tokyo 113-0033, Japan
}

\begin{abstract}
There are three major axes to describe the evolution of galaxies, namely, time (redshift), space (environment) and mass (stellar mass). In this article, one topic each will be presented along these axes. (1) Based on the Subaru wide-field (30') optical imaging of two distant clusters $(z=0.55$ and 0.83$)$, we show the large scale structures in galaxy distributions on a scale greater than $10 \mathrm{Mpc}$, which serve as an evidence for hierarchical growth of rich clusters of galaxies through assembly of surrounding groups. (2) Based on the deep NIR imaging of high- $z$ clusters at $z \sim 1$ and 1.5, we show massive galaxies in clusters have assembled most of their mass by $z=1.5$, which is earlier than the hierarchical model predictions. (3) Based on the Subaru deep and wide optical imaging of Subaru/XMM-Newton Deep Survey Field, we show a deficit of red and faint galaxies and a lack of blue massive galaxies in the high density regions at $z \sim 1$, which suggest down-sizing in galaxy formation as apparently opposed to the CDM-based bottom-up scenario.
\end{abstract}

\section{Space: Panoramic Imaging and Spectroscopy of Cluster Evolution with Subaru (PISCES)}

First of all, along the space axis, we present panoramic imaging of two distant clusters taken with Subaru wide-field optical camera Suprime-Cam which provides $34^{\prime} \times 27^{\prime}$ field of view corresponding to $15 \times 12 \mathrm{Mpc}^{2}$ at $z=0.8$. This project called PISCES (Panoramic Imaging and Spectroscopy of Cluster Evolution with Subaru) has started since 2003, and we aim to target 10-15 X-ray selected distant clusters in total at $0.4 \lesssim z \lesssim 1.3$, in good coordination with $A C S / H S T$, $X M M$, and Chandra observations. The goals of this programme is to map out the large scale structures around clusters to trace the cluster assembly history and then to look into the galaxy properties as a function of environment along the structures to directly identify the environmental effects acting on galaxies during their assembly to higher density regions. This unique project is currently underway and just a preliminary result on the large scale structures is shown 

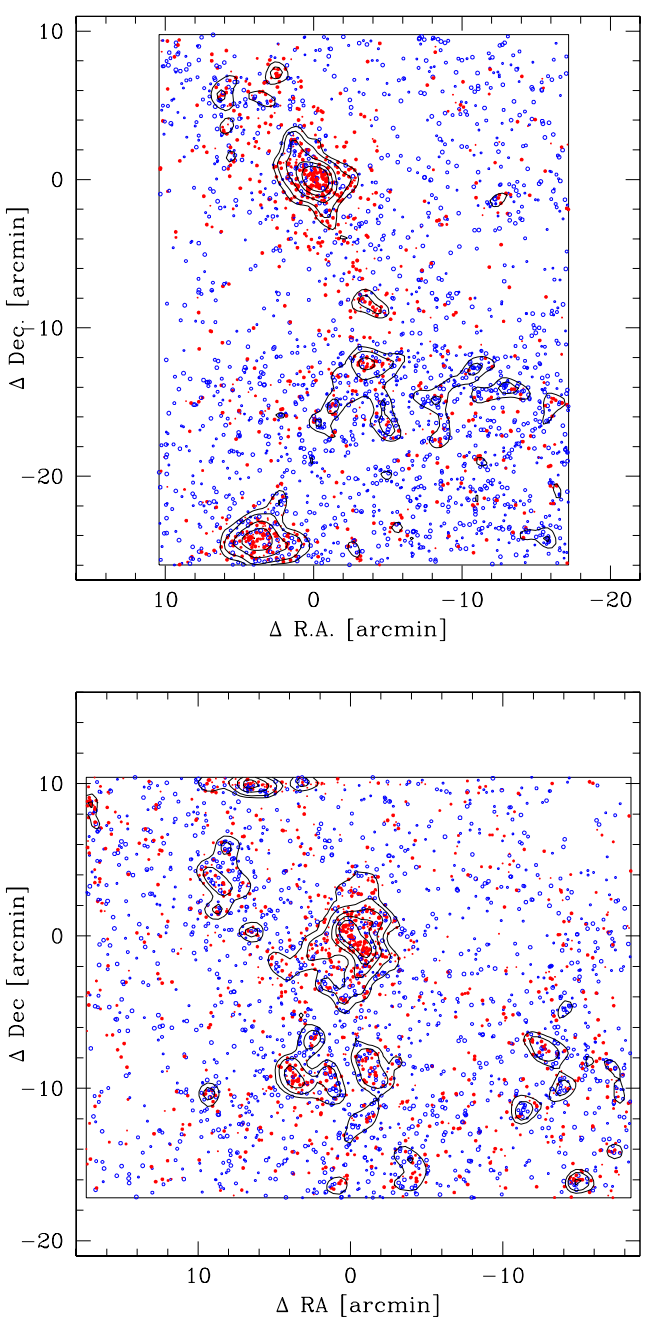

Fig. 1. The panoramic maps of CL0016+16 cluster $(z=0.55)$ and RXJ0152.7-1357 $(z=0.83)$ are presented on the top and the bottom panels (10 arcminutes corresponds to $3.8 \mathrm{Mpc}$ and $4.6 \mathrm{Mpc}$, respectively). Using photometric redshift technique based on multi-colour data $\left(B V R i^{\prime} z^{\prime}\right.$ and $V R i^{\prime} z^{\prime}$, respectively), only photometric member candidates are plotted selected with redshift cuts of $0.47 \leq z \leq 0.6$ and $0.75 \leq z \leq 0.9$, respectively. Contours show local 2-D number density of galaxies at $1.5,2,3,4,5 \sigma$ above the mean density. Coordinates are shown relative to the centre of the main cluster. Large scale filamentary structures $(>10 \mathrm{Mpc})$ are seen in both clusters.

in Fig. 1. These two rich clusters at $z=0.55$ and 0.83 are imaged in multi optical bands with Suprime-Cam, and photometric redshifts [13] have been applied to efficiently remove foreground/background contaminations and to isolate the 
cluster member candidates (cf., [14]). Many substructures are now clearly seen around the main body of the clusters which tend to be aligned in filamentary structures extending to $>10 \mathrm{Mpc}$ scale across. Although these structures should be confirmed spectroscopically later on, these already provide good evidence for cluster assembly in the hierarchical Universe.

[14] have presented the environmental dependence of galaxy colours along the filamentary structures around the A851 cluster $(z=0.41)$, and have shown that the galaxy colour changes rather sharply at relatively low density regions like galaxy groups along the filaments well outside of the cluster core. Together with the similar findings in the local Universe [18][9]. the environmental effects that truncate star formation are not the cluster specific phenomena such as rampressure stripping [1] but are found to be much wider spread into low density regions. It is important to extend this analysis to higher redshifts as the galaxy environment is expected to change dramatically during the course of vigorous assembly, which is probably related to the appearence of morphology-density relation [8].

\section{Time: Stellar mass assembly of massive galaxies in high- $z$ clusters}

Secondly, along the time axis, we present the stellar mass functions of galaxies in high- $z$ clusters constructed from deep near-infrared imaging ( $J$ and $\left.K_{s}\right)$ (Fig. 2). The stellar mass function of galaixes derived from the $K$-band observations is a good tracer of mass assembly history of galaxies (eg., [10][3]). We have combined two $z \sim 1$ clusters (3C336 and Q1335+28) [16], and five $z \sim 1.5$ clusters (Q0835+580, Q1126+101, Q1258+404, Q0139-273, and Q2025-155) [20][21][4] to increase statistics. We have subtracted the control field counts taken from the literature [23][24][4]. Applying the same technique described in [16], we construct the field-subtracted stellar mass functions of galaxies in high- $z$ clusters, primarily using $K_{s}$-band flux and also using $J-K_{s}$ colour as a measure of the $\mathrm{M} / \mathrm{L}$ ratio. As shown, little evolution is observed since $z=1.5$ to the presentday (2MASS clusters [2]), indicating that the mass assembly on galaxy scale is largely completed by $z \sim 1.5$ in the cluster environment. This epoch of mass assembly of massive galaxies is earlier than the prediction of the hierarchical models as shown for comparison [19].

It is also interesting to trace back the stellar mass to even higher redshift to firstly identify the epoch of assembly of massive galaxies where they start to break down into pieces. Recent deep NIR observations in fact start to enter such formation epoch (eg., [7][22][6]), and this aspect will be further extended by on-going and future space missions such as SIRTF and Astro-F.

\section{Mass: Down-sizing in galaxy formation seen at $z \sim 1$}

Finally, along the mass axis, we present how the star formation history depends on the mass of the galaxies at $z \sim 1$ based on the unique Suprime-Cam imaging 


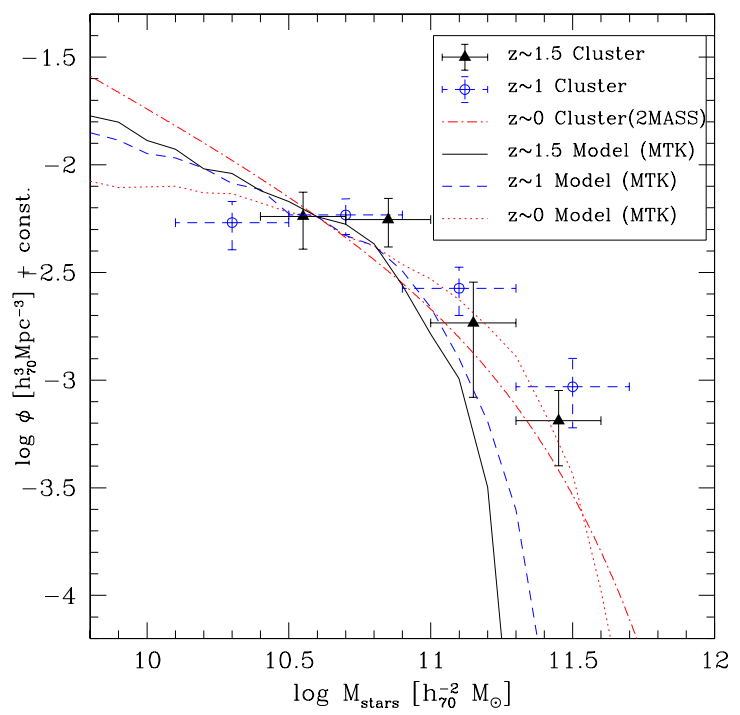

Fig. 2. Stellar mass functions of galaxies in clusters as a fuction of redshift. The Kennicutt's initial mass function [11] is used to scale the stellar mass. The open diamonds and the filled triangles show the stellar mass functions for $z \sim 1$ and $z \sim 1.5$ clusters, respectively, which are compared to the local counterpart from the 2MASS survey (dotdashed curve). All the curves and the data points are normalised at $5 \times 10^{10} \mathrm{M}_{\odot}$ so as to have the same amplitude. The theoretical predictions from a semi-analytic model [19] are presented for comparison, which are made for galaxies in the haloes whose circular velosities are greater than $1000 \mathrm{~km} / \mathrm{s}$ at each epoch. The mass assembly of massive galaxies in the real Universe is much faster than the hierarchical model prediction.

data $\left(B R i^{\prime} z^{\prime}\right)$ on the Subaru/XMM-Newton Deep Survey (SXDS) Field. Our data are both sufficiently deep $\left(z_{A B}^{\prime}=25,6-10 \sigma\right)$ and wide $\left(1.2 \mathrm{deg}^{2}\right)$, which enable us for the first time to investigate the photometric properties of statistical sample of galaxies at $z \sim 1$ down to $\sim M^{*}+3$ with respect to the passive evolution. We first identify five $z \sim 1$ high density regions by applying colour cuts at $1.7<R-z^{\prime}<2.0$ and $0.8<i^{\prime}-z^{\prime}<1.1$, which correspond to the colours of passively evolving galaxies at $z \sim 1[12]$. We then combine these five regions (amounting to $141 \mathrm{arcmin}^{2}$ in total) and subtract off the low density regions at $z \sim 1$ (scaled to the same area) on the colour-magnitude diagram to isolate only $z \sim 1$ galaxies in a statistical sense, since the both regions are expected to have the same amount of foreground/background contaminations.

Thus constructed field-corrected colour-magnitude diagram for $z \sim 1$ galaxies in high density regions is shown in Fig. 3. Most striking feature of this diagram is that there are two distinct populations, 'bright+red' and 'faint+blue'. More precisely, we show a deficit of red and faint galaxies below $M^{*}+2$ or $10^{10} \mathrm{M}_{\odot}$ in stellar mass and a lack of blue massive galaxies beyond $M^{*}-0.5$ or $8 \times 10^{10} \mathrm{M}_{\odot}$ 


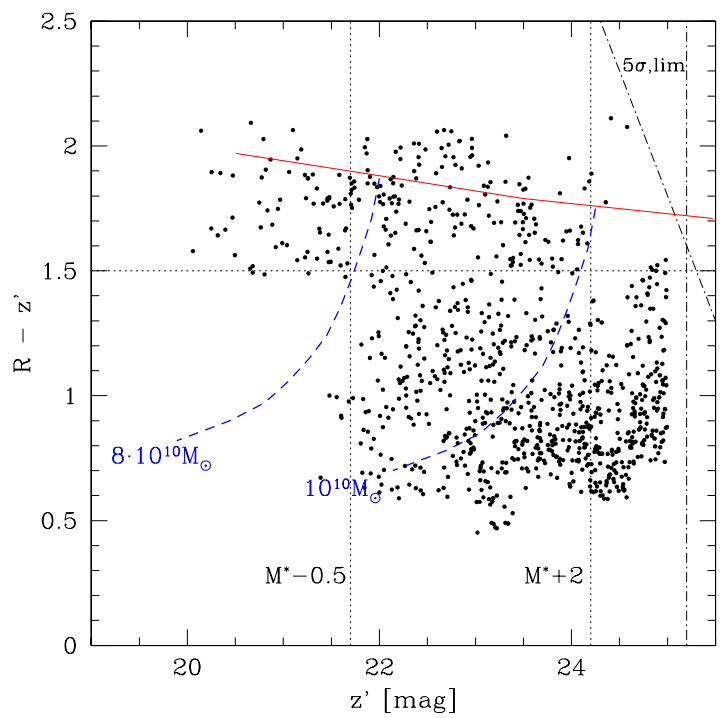

Fig. 3. Field-corrected colour-magnitude diagram for the $z \sim 1$ galaxies in high density regions [17]. The solid line show the expected location of colour-magnitude sequence at $z \sim 1$ assuming a passive evolution with $z_{\text {form }}=5$ [12]. The deficit of both blue galaxies at the bright/massive end and the deficit of red galaxies at the faint/less-massive end are both clearly identified.

in stellar mass. This bimodality in colour-magnitude distributions can be also seen in the colour-dependent stellar mass function shown in Fig. 4. Red galaxies tend to be bright and show a bell-like shape, while the blue galaxies tend to be faint and show a much steeper faint-end slope. These results indicate the down-sizing in galaxy formation [5], where star formation and mass assembly in massive galaxies take place early in the Universe, while the star formation seems to be progressively shifted towards less massive systems as the Universe ages. Next obvious important step is to directly detect the brightening of such break luminosity or mass with increasing redshift [15].

We have assumed the cosmological parameters of $\left(H_{0}, \Omega_{m}, \Omega_{\Lambda}\right)=(70,0.3$, $0.7)$, throughout this paper.

We thank Drs. Ian Smail and Eric Bell for useful discussion. We also acknowledge to the SXDS team (Kaz Sekiguchi et al.). This work was financially supported in part by a Grant-in-Aid for the Scientific Research (No. 15740126) by the Japanese Ministry of Education, Culture, Sports and Science.

\section{References}

1. Abadi, M. G., Moore, B., Bower, R. G.: MNRAS, 308, 947 (1999) 


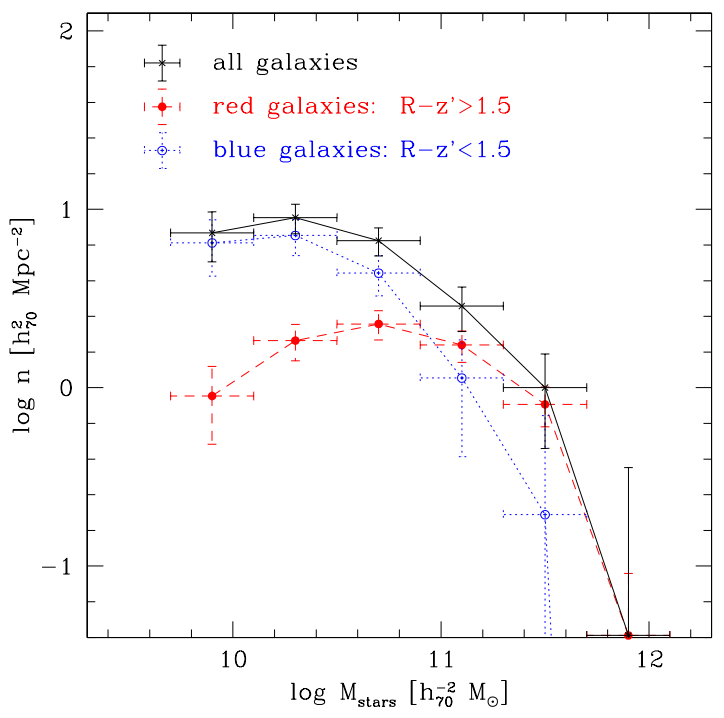

Fig. 4. Colour-dependent stellar mass functions of the $z \sim 1$ galaxies in high density regions [17]. The stellar masses are scaled using the Kennicutt's IMF [11].

2. Balogh, M. L., Christlein, D., Zabludoff, A. I., Zarisky, D.: ApJ, 557, 117 (2001)

3. Baugh, C. M., Benson, A. J., Cole, S., Frenk, C. S., Lacey, C.: astro-ph/0203051 (2002)

4. Best, P. N., Lehnert, M. D., Miley, G. K., Röttgering, H. J. A.: MNRAS, 343, 1 (2003)

5. Cowie, L. L., Songaila, A., Hu, E. M., Cohen, J. G.: AJ, 112, 839 (1996)

6. Daddi, E., et al.: astro-ph/0308456 (2003)

7. Dickinson, M. Papovich, C., Ferguson, H. C., Budavari, T.: ApJ, 587, 25 (2003)

8. Dressler, A.: ApJ, 236, 351 (1980)

9. Gómez, P. L., et al.: ApJ, 584, 210 (2003)

10. Kauffmann, G., Charlot, C.: MNRAS, 297, 23 (1998)

11. Kennicutt, R. C. Jr.: ApJ, 272, 54 (1983)

12. Kodama, T., Arimoto, N., Barger, A. J., Aragón-Salamanca, A.: A\&A, 334, 99 (1998)

13. Kodama, T., Bell, E. F., Bower, R. G.: MNRAS, 302, 152 (1999)

14. Kodama, T., Smail, I., Nakata, F., Okamura, S., Bower, R. G.: ApJ, 562, L9 (2001)

15. Kodama, T., Bower, R. G.: MNRAS, 321, 18 (2001)

16. Kodama, T., Bower, R. G.: MNRAS, 346, 1 (2003)

17. Kodama, T., Yamada, T., et al.: submitted (2003)

18. Lewis, I., et al.: MNRAS, 334, 673 (2002)

19. Nagashima, M., Yoshii, Y., Totani, T., Gouda, N.: ApJ, 578, 675 (2002)

20. Hall, P. B., Green, R. F., Cohen, M.: ApJS, 119, 1 (1998)

21. Hall, P. B., et al.: AJ, 121, 1840 (2001)

22. Rudnick, G., et al.: astro-ph/0307149 (2003)

23. Saracco, P., et al.: A\&A, 349, 751 (1999)

24. Saracco, P., et al.: A\&A, 375, 1 (2001) 